\title{
Hematopoietic \& mesenchymal - the two lineages of bone marrow stem cells
}

\section{Introduction}

Bone marrow is the flexible tissue found in the interior of bones In humans, bone marrow in large bones produces new blood cells. On average, bone marrow constitutes $4 \%$ of the total body mass of humans; in adults weighing $65 \mathrm{~kg}$, bone marrow accounts for approximately $2.6 \mathrm{~kg}$. The hematopoietic compartment of bone marrow produces approximately 500 billion blood cells per day, which use the bone marrow vasculature as a conduit to the body's systemic circulation. ${ }^{1,2}$

The bone marrow consists of multipotent uncommitted stem cells and also committed stem cells. The pluripotent cells can give rise to many varieties of cells but committed stem cell can give rise to only one variety to which it is committed. Committed stem cells originate from pluripotent stem cells. Two types of stem cells present are Hematopoietic stem cells and Mesenchymal stem cells.

\section{Hematopoietic stem cells}

Hematopoietic stem cells (HSCs), are multipotent stem cells that give rise to all the cell types of myeloid and lymphoid lineages. There are various markers that can be used to detect HSCs. Ex. CD34, CD59, Thy1, CD38, C-kit, lin etc. ${ }^{3}$ HSCs have some functional characteristics. They can renew and differentiate, can be mobilized from bone marrow to blood and reverse also. It also can undergo for programmed cell death. As stem cells, HSCs are defined by their ability to replenish all blood cell types (Multipotency) and their ability to self-renew. ${ }^{4}$ It is known that a small number of HSCs can expand to generate a very large number of daughter HSCs. This phenomenon is used in bone marrow transplantation, when a small number of HSCs reconstitute the hematopoietic system. ${ }^{5}$ This indicates that, subsequent to bone marrow transplantation, symmetrical cell divisions into two daughter HSCs must occur.

Stem cell self-renewal is thought to occur in the stem cell niche in the bone marrow, and it is reasonable to assume that key signals present in this niche will be important in self-renewal. Understanding the ability of HSC to replenish themselves will eventually allow the generation of expanded populations of HSC in vitro that can be used therapeutically.

HSCs are heterogeneous in nature. The stem cell compartment can be split into three categories as - Balanced (Bala) HSC repopulate peripheral white blood cells. Myeloid-biased (My-bi) HSC give rise to too few lymphocytes lymphoid-biased (Ly-bi) HSC generate too few myeloid cells. These are three types of HSC, and they do not represent stages of differentiation. ${ }^{7}$ Rather, these are the three classes of HSC, each with an epigenetically fixed differentiation program. Functional assay is possible for these cells. A cobblestone areaforming cell (CAFC) assay is a cell culture-based empirical assay. When plated onto a confluent culture of stromal feeder layer, a fraction of HSCs creep between the gaps (even though the stromal cells are touching each other) and eventually settle between the stromal cells and the substratum (here the dish surface) or trapped
Volume 2 Issue 4 - 2017

\author{
Arati Adhe-Rojekar,' Mohit V Rojekar ${ }^{2}$ \\ 'Hinduja National Hospital and Research Center, India \\ ${ }^{2}$ Rajiv Gandhi Medical College, India
}

Correspondence: Mohit V Rojekar, Rajiv Gandhi Medical College, Kalwa, Thane, India, Email drmohi44@gmail.com

Received: February 28, 2017 | Published: April 24, 2017

in the cellular processes between the stromal cells. Emperipolesis is the in vivo phenomenon in which one cell is completely engulfed into another; on the other hand, when in vitro, lymphoid lineage cells creep beneath nurse-like cells, the process is called pseudoemperipolesis. This similar phenomenon is more commonly known in HSC field by the cell culture terminology cobble stone area-forming cells (CAFC), which means areas of cluster of cells that look dull cobblestone-like under phase contrast microscopy, compared to the other HSCs, which are refractile. ${ }^{8,9}$ This happens because the cells that are floating loosely on top of the stromal cells are spherical and thus refractile. However, the cells that creep beneath the stromal cells are flattened and, thus, not refractile.

HSCs have characteristic mobility. HSCs have a higher potential than other immature blood cells to pass the bone marrow barrier, and, thus, may travel in the blood from the bone marrow in one bone to another bone. If they settle in the thymus, they will develop into $\mathrm{T}$ cells. In the case of fetuses and other extramedullary hematopoiesis, HSCs may also settle in the liver or spleen and develop. This ability is the reason why HSCs may be harvested directly from the blood. ${ }^{5}$

Hematopoietic stem cell transplantation (HSCT) is the transplantation of multipotent hematopoietic stem cell or blood, usually derived from bone marrow, peripheral blood stem cells, or umbilical cord blood. ${ }^{10}$ Hematopoietic stem cell transplantation remains a risky procedure with many possible complications; it has traditionally been reserved for patients with life-threatening diseases. While occasionally used experimentally in nonmalignant and nonhematologic indications such as severe disabling autoimmune disease and cardiovascular disease, the risk of fatal complications appears too high to gain wider acceptance. There are many diseases which could be treated using stem cells like Sickle-cell disease, Myelodysplastic syndrome, Meuroblastoma, Lymphoma, Ewing's Sarcoma, Desmoplastic small round cell tumor, Chronic granulomatous disease, Hodgkin's disease.

More recently non-myeloablative, or so-called "mini transplant," procedures have been developed that require smaller doses of preparative chemo and radiation. This has allowed HSCT to be conducted in the elderly and other patients who would otherwise be considered too weak to withstand a conventional treatment regimen. 


\section{Mesenchymal stem cells}

The bone marrow stroma contains mesenchymal stem cells (MSCs), also called marrow stromal cells. These are multipotent stem cells that can differentiate into a variety of cell types. MSCs have been shown to differentiate, in vitro or in vivo, into osteoblasts chondrocytes, myocytes, adipocytes and beta pancreatic islet cells. MSCs can also trans-differentiate into neuronal cells. ${ }^{11,12}$

MSCs, are multipotent stem cells that can differentiate into a variety of cell types including Osteoblasts (bone cells), Chondrocytes (cartilage cells) and Adipocytes (fat cells). This has been shown in ex vivo cultures and in vitro or in vivo.

There are various sources of MSCs. The youngest, most primitive MSC's can be obtained from the umbilical cord tissue, namely Wharton's jelly and the umbilical cord blood. However the MSC's are found in much higher concentration in the Wharton's jelly compared to the umbilical cord blood, which is a rich source of hematopoeitic stem cells. The umbilical cord MSCs have more primitive properties than other adult MSCs obtained later in life, which might make them a useful source of MSCs for clinical applications. ${ }^{13,14}$ An extremely rich source for mesenchymal stem cells is the developing tooth bud of the mandibular third molar. The stem cells eventually form enamel, dentin, blood vessels, dental pulp, nervous tissues, including a minimum of 29 different unique end organs. ${ }^{15}$ These stem cells have been shown capable of producing hepatocytes. Additionally, amniotic fluid has been shown to be a very rich source of stem cells. As many as 1 in 100 cells collected from and genetic amniocentesis has been shown to be a pluripotent mesenchymal stem cell. Adipose tissue is one of the richest sources of MSC's. Adipose stem cells are currently actively being researched in clinical trials for treatment in a variety of diseases. ${ }^{16,17}$

MSCs are of intense therapeutic interest because they represent a population of cells with the potential treat a wide range of acute and degenerative diseases. MSCs are advantageous over other stem cells types for several reasons. First, they avoid the ethical issues that surround embryonic stem cell research. Second, repeated studies have found that human MSCs are immuno-privileged, and therefore, represent an advantageous cell type for allogenic transplantation, reducing the risks of rejection and complications of transplantation. Recently, there have also been significant advances in the use of autologous MSCs to regenerate human tissues, including cartilage and meniscus, tendons, and bone fractures. ${ }^{18}$

\section{Acknowledgements}

None.

\section{Conflict of interest}

The author declares no conflict of interest.

\section{References}

1. Fliedner TM, Graessle D, Paulsen C, et al. Structure and function of bone marrow hemopoiesis: mechanisms of response to ionizing radiation exposure. Cancer Biother Radiopharm. 2002;17(4):405-426.
2. Vunjak-Novakovic G, Tandon N, Godier A, et al. Challenges in cardiac tissue engineering. Tissue Eng Part B Rev. 2010;16(2):169-187.

3. Seita J, Weissman IL. Hematopoietic stem cell: Self-renewal versus differentiation. Wiley Interdiscip Rev Syst Biol Med. 2010;2(6):640-653.

4. Kondo M. Lymphoid and myeloid lineage commitment in multipotent hematopoietic progenitors. Immunol Rev. 2010;238(1):37-46.

5. Ma H, Young M, Yang Y. Hematopoietic stem cells. Stem Cell. 2015;6(1):1-4

6. Morrison SJ, Spradling AC. Stem cells and niches: mechanisms that promote stem cell maintenance throughout life. Cell. 2008;132(4):598611.

7. Muller-Sieburg CE, Sieburg HB. The GOD of hematopoietic stem ce1ls: a clonal diversity model of the stem cell compartment. Cell cycle. 2006;5(4):394-398.

8. Neben S, Anklesaria P, Greenberger J, et al. Quantitation of murine hematopoietic stem cells in vitro by limiting dilution analysis of cobblestone area formation on a clonal stromal cell line. Exp Hematol. 1993;21(3):438-443.

9. Frisch BJ, Calvi LM. Hematopoietic stem cell cultures and assays. $\mathrm{Me}$ thods in molecular biology. 2014;1130:315-324.

10. Eaves CJ. Hematopoietic stem cells: concepts, definitions, and the new reality. Blood. 2015;125(17):2605-2613.

11. Prockop DJ. Marrow stromal cells as stem cells for nonhematopoietic tissues. Science. 1997;276(5309):71-74.

12. Malgieri A, Kantzari E, Patrizi MP, et al. Bone marrow and umbilical cord blood human mesenchymal stem cells: state of the art. Int J Clin Exp Med. 2010;3(4):248-269.

13. Kalaszczynska I, Ferdyn K. Wharton's jelly derived mesenchymal stem cells: future of regenerative medicine? Recent findings and clinical significance. Biomed Res Int. 2015;2015:11.

14. Kim DW, Staples M, Shinozuka K, et al. Wharton's jelly-derived mesenchymal stem cells: Phenotypic characterization and optimizing their therapeutic potential for clinical applications. Int J Mol Sci. 2013;14(6):11692-11712.

15. Volponi AA, Pang Y, Sharpe PT. Stem cell-based biological tooth repair and regeneration. Trends in Cell Biology. 2010;20(12):715-722.

16. Ullah I, Subbarao RB, Rho GJ. Human mesenchymal stem cells - current trends and future prospective. Bioscience Reports. 2015;35(2):e00191.

17. Savickiene J, Treigyte G, Baronaite S, et al. Human amniotic fluid mesenchymal stem cells from second- and third-trimester amniocentesis:Differentiation potential, molecular signature, and proteome analysis. Stem Cells Int. 2015;2015:15.

18. Saeed H, Ahsan M, Saleem Z, et al. Mesenchymal stem cells (MSCs) as skeletal therapeutics - an update. J Biomed Sci. 2016;23:41. 